\title{
Novel mutations, including the second most common in Japan, in the $\beta$-hexosaminidase $\alpha$ subunit gene, and a simple screening of Japanese patients with Tay-Sachs disease
}

\begin{abstract}
Two novel mutations of the $\beta$-hexosaminidase $\alpha$ subunit gene were identified in Japanese patients with the infantile form of Tay-Sachs disease. One mutation was a one-base deletion at nt613C, which generated a stop codon at two codons downstream, in three unrelated patients. The other mutation was a one-base substitution of G-to-A at IVS $5,+1$, which caused a splicing abnormality, in one patient. A missense mutation of R170W, which has already been reported in other ethnic groups, was also newly identified in one patient. In 1993, the most common mutation (IVS $5,-1 \mathrm{G} \rightarrow \mathrm{T}$ ) in Japanese patients with Tay-Sachs disease was reported as the major mutation in Japan accounting for $80 \%$ of 56 mutant alleles from 28 unrelated patients. The deletion of nt613C was the second most common mutation, accounting for $5 \%$ of the mutant alleles. The previously reported mutation IVS $5,-1 \mathrm{G} \rightarrow \mathrm{T}$ and the nt613C deletion found in this study together accounted for $85 \%$ of the mutations causing Tay-Sachs disease among Japanese. Since these two mutations were located in or close to exon 6 and since they abolish Fok I (IVS 5, -1G $\rightarrow$ T) and Sfa NI (nt613C deletion) restriction sites, respectively, they were screened rapidly by single polymerase chain reaction followed by digestion with these enzymes.
\end{abstract}

Key words Tay-Sachs disease $\cdot$ Hexosaminidase $\alpha$ subunit gene - Molecular diagnosis - The two major mutations . Mutation screening

A. Tanaka $(\bowtie) \cdot$ M. Fujimaru $\cdot$ G. Isshiki

Department of Pediatrics, Osaka City University Medical School, 1-4-3 Asahi-machi, Abeno-ku, Osaka 545-8585, Japan

Tel. +81-6-6645-3816; Fax +81-6-6636-8737

e-mail: akemi-chan@msic.med.osaka-cu.ac.jp

K. Choeh

Department of Pediatrics, Eulji Medical College Hospital, Taejon, Korea

\section{Introduction}

Tay-Sachs disease is an autosomal recessive disorder that primarily affects the central nervous system. It is caused by mutations in the gene encoding the $\alpha$ subunit of $\beta$ hexosaminidase A (Hex A), a lysosomal enzyme composed of $\alpha$ and $\beta$ polypeptides. Hex A requires the assistance of GM2 activator protein for hydrolysis of the lipophilic GM2ganglioside in the hydrophilic environment of the lysosome. Mutations in the $\beta$ subunit and GM2 activator protein, respectively, result in the two other GM2-gangliosidoses, known as Sandhoff disease and activator protein deficiency. Deficient catabolism and abnormal accumulation of GM2ganglioside is a common feature in all GM2-gangliosidoses and, consequently, they all exhibit similar clinical symptoms.

Seventy-eight mutations in the $\alpha$ subunit gene had been described in the literature by 1997 (Myerowitz, 1997). Some mutations are commonly found in ethnically or geographically isolated populations. A 4-bp insertion in exon 11 occurs in $80 \%$ of infantile Ashikenazi Jewish patients (Myerowitz and Costigan, 1988), while a splice junction mutation at the donor splice site of exon 12 is found in $15 \%$ of patients in the same population (Arpaia et al. 1988; Myerowitz, 1988; Ohno and Suzuki, 1988). A 7.6-kb deletion, including all of exon 1 and parts of the flanking sequences, is the major mutation causing Tay-Sachs disease in the French Canadian population (Myerowitz and Hogikyan, 1987). We have earlier reported a mutation, IVS $5,-1 \mathrm{G} \rightarrow \mathrm{T}$, accounting for $80 \%$ of the mutant alleles among Japanese patients with Tay-Sachs disease, to be the major mutation in Japan (Tanaka et al. 1993). The five additional disease-causing mutations that we reported subsequently were all limited to single alleles in 28 unrelated patients (Tanaka et al. 1994). In this report, we describe two novel mutations and one additional mutation in the $\alpha$ subunit gene, one of which is the second most common mutation in Japanese patients. We also describe a simple and rapid screening procedure for the two most common mutations. 


\section{Patients and methods}

Subjects and fibroblast culture

Five unrelated patients were studied (subjects 2, 4, 5, 7, and 8 ). We had determined ealier that four of them (subjects 2 , 4,5 , and 7 ) had the major mutation (IVS $5,-1 \mathrm{G} \rightarrow \mathrm{T}$ ) in one allele (Tanaka et al. 1993), but the mutations in subject 8 were completely unknown. Subjects 1,3 , and 6 , already known to be homoallelic with the major mutation (IVS 5, $-1 \mathrm{G} \rightarrow \mathrm{T}$ ), were used to test the simple screening procedure. The parents of the eighth patient (subject 8) were consanguineous and the family was from the Okinawa Islands, where the ethnic background of the population is different from that in other parts of Japan.

Skin fibroblasts were maintained in Eagle's MEM with $10 \%$ fetal calf serum at $37^{\circ} \mathrm{C}$, under $5 \% \mathrm{CO}_{2}$ and $100 \%$ humidity.

\section{Mutation analysis}

Genomic DNA was extracted by the standard method (Shambrook et al. 1989). Each exon and the flanking regions were amplified with appropriate primers, according to Triggs-Raine et al. (1991).

Amplified exons were submitted to single-strand conformation polymorphism (SSCP) analysis using the Phast System (Pharmacia Biotech Japan, Tokyo) in 12.5\% polyacrylamide gel (PhastGel homogeneous 12.5) with PhastGel Native Buffer Strip and stained with silver with a PhastGel Silver Kit.

Each polymerase chain reaction (PCR) fragment that gave an abnormal SSCP pattern was subcloned with a TA Cloning Kit (Invitrogen, San Diego, CA, USA) and sequenced by the capillary sequencer ABI PRISM 310 Genetic Analyzer (Perkin Elmer Japan/ABI, Chiba, Japan) with a dRhodamin Terminator Cycle Sequencing Kit from the same company.

Simple screening procedure for the two major mutations

The sequence surrounding exon 6 was amplified from genomic DNA extracted by the standard method with primers, 5' -TGAAACCGGAGAGACTGTGATG-3' (sense) and 5'-GCCACAGCCAGATTCAGACATTG-3' (antisense), by 35 cycles of 1 -min denaturation at $94^{\circ} \mathrm{C}, 2-$ min annealing at $58^{\circ} \mathrm{C}$, and 3 -min extension at $72^{\circ} \mathrm{C}$. The product was digested with either Fok I (GGATG $\mathrm{N}_{9} / \mathrm{N}_{13}$ ) or $S f a \mathrm{NI}\left(\mathrm{GCATC} \mathrm{N}_{5} / \mathrm{N}_{9}\right)$ at $37^{\circ} \mathrm{C}$ for 3-4h, electrophoresed in $2 \%$ agarose gel, and photographed.

\section{Results}

Mutation analysis

At least three bands, the fourth of which was not visible, were detected by the SSCP analysis of exon 6 in subjects 2,

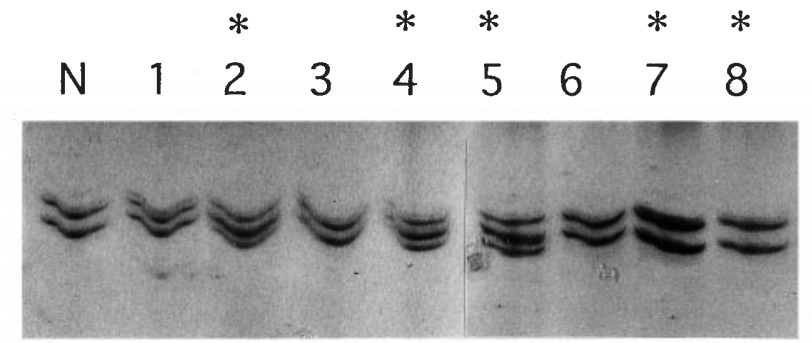

Fig. 1 Single-strand conformation polymorphism (SSCP) analysis of the polymerase chain reaction (PCR) fragment of exon 6 and flanking regions. Lane $N$, Normal control; lanes $1-8$, subjects $1-8$, respectively, with Tay-Sachs disease. Subjects 2, 4, 5, 7, and 8 (asterisked) have unknown alleles. At least three bands were visible in lanes 2, 4, and 5

4, and 5 (Fig. 1). Upon subcloning and sequencing of exon 6 , a deletion of nt613C, which generated a stop codon at two codons downstream, was found in one allele in these subjects (Fig. 2a). The deletion abolished an Sfa NI site in the normal sequence. All of these patients were heteroallelic with this deletion. The allele containing this mutation appeared to generate no mRNA, since reverse transcription (RT)-PCR detected only the abnormally short mRNA without exon 6 derived from the other allele with IVS 5, $-1 \mathrm{G} \rightarrow \mathrm{T}$ (data not shown).

A mutation of IVS $5,+1 \mathrm{G} \rightarrow$ A was found by sequence analysis in subject 7 in the heteroallelic state (Fig. 2b). A missense mutation of R170W (nt508, C $\rightarrow$ T) was identified in subject 8 in the homoallelic state and this mutation abolished a normally present $M s p$ I site (CCGG $\rightarrow$ CTGG) (Fig. 3a,b).

Simple screening for the two major mutations

IVS $5,-1 \mathrm{G} \rightarrow \mathrm{T}$ (the major mutation) was readily identified because the PCR fragment of exon 6 from the normal allele was cut into two fragments, 148 bp and 63 bp, by Fok I, while the fragment from the mutant allele was resistant to Fok I (211 bp) (Fig. 4b). Patients who are homoallelic (Fig. $4 a$, lanes 1,3 , and 6 , or subjects 1,3 , and 6 ) and heteroallelic (Fig. 4a, lanes 2, 4, and 5, or subjects 2, 4, and 5) for IVS 5, $-1 \mathrm{G} \rightarrow \mathrm{T}$ were unequivocally identified. Similarly, the same PCR fragment from the normal allele was digested into 113-bp and 98-bp fragments by Sfa NI, while the fragment from the allele containing the nt613C deletion (the second major mutation) was resistant (Fig. 5b). Subjects 2, 4, and 5 had the nt613C deletion (Fig. 5a, lanes 2, 4, and 5) in one allele.

\section{Discussion}

Ethnically and geographically isolated populations tend to harbor certain mutations at high frequency and even uniquely. Such mutations are important not only for diagnosis but also for studies of human population genetics. Japan is no exception. The one-base substitution of nt727, 
a

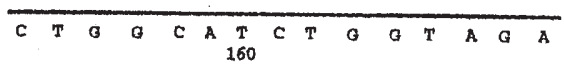

Normal

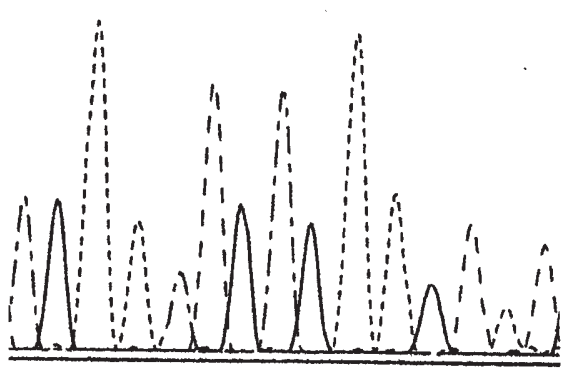

$\triangle 613 C$

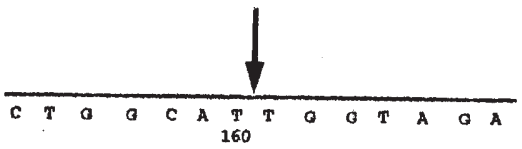

Fig. 2 a Sequence chart of the mutation of one-base deletion of $613 \mathrm{C}$ in subject 2 (bottom) and the normal sequence (top). b Sequence chart of the mutation of one-base substitution of G-to-A at +1 of intron 5 in

a

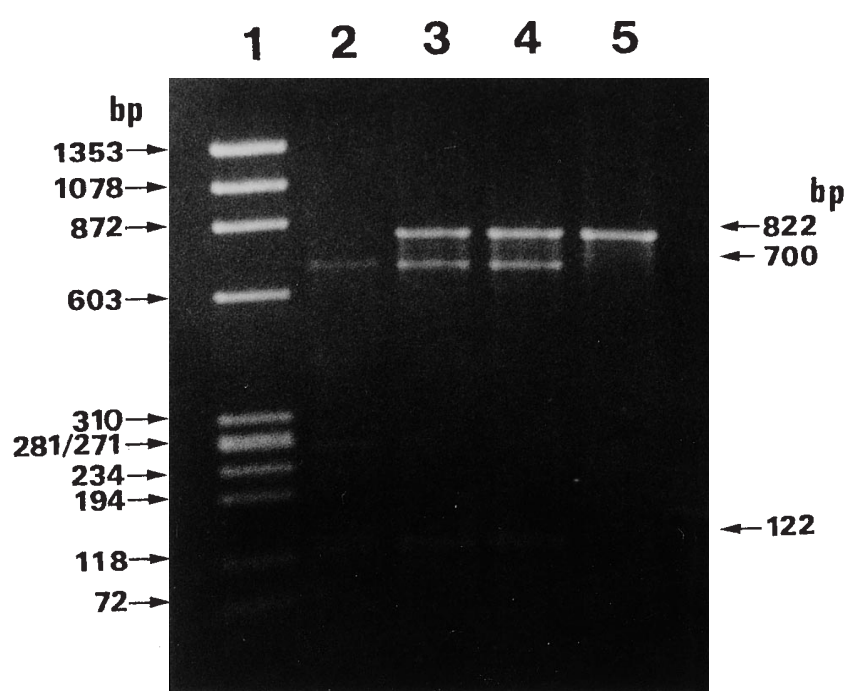

Fig. 3 a Agarose gel electrophoresis of $M s p$ I-digested PCR fragment of genomic DNA from intron 3 to intron 5 in the eighth patient (subject 8 ) and his parents. Lane 1, Molecular size marker; lane 2, normal control; lane 5, the patient; lanes 3 and 4 the patient's father and mother, respectively. The patient was homoallelic for the mutant allele b
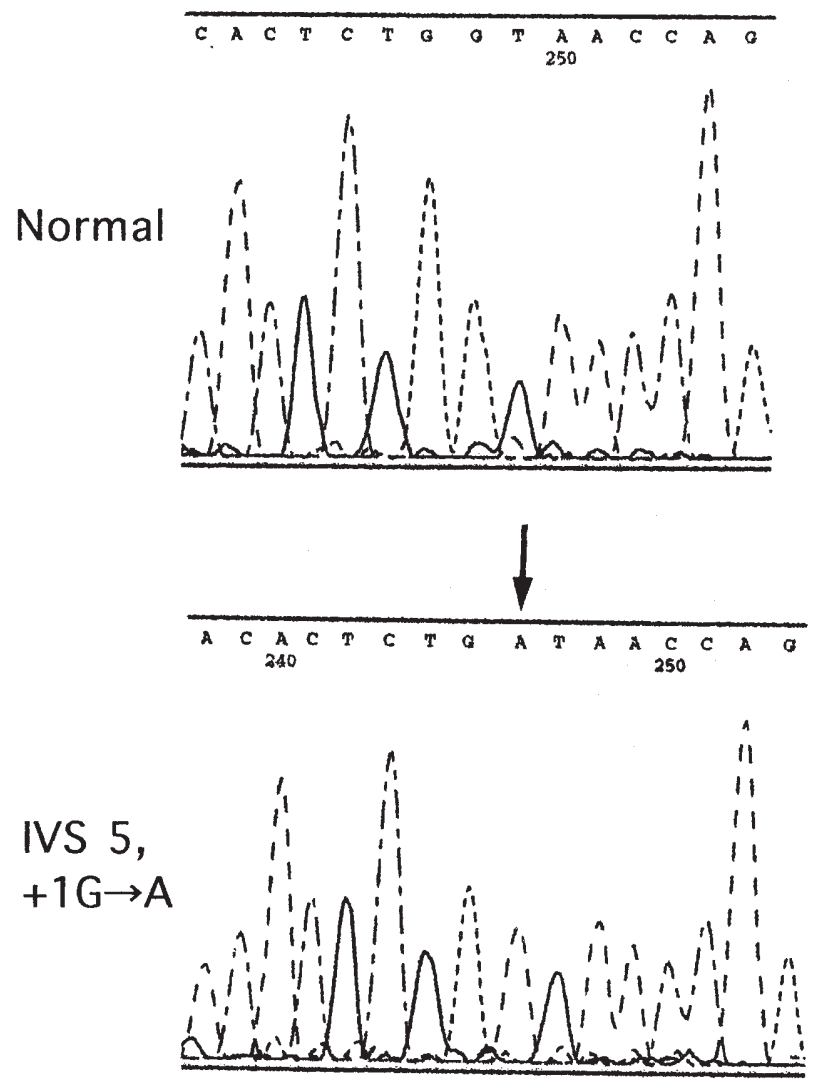

subject 7 (bottom) and the normal sequence (top). Arrows indicate the mutations

b

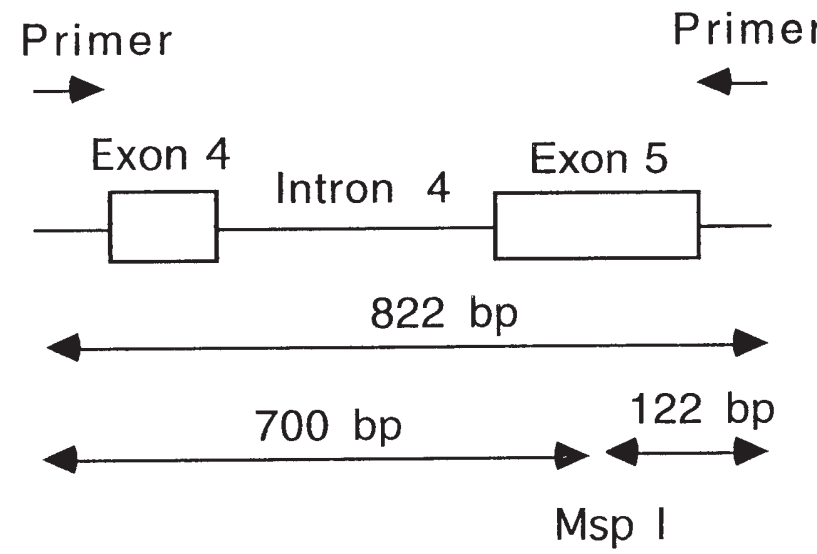

Normal: CCGG

Mutant: $\quad$ CTGG

and both parents carried the same mutation. b Schematic illustration of the restriction site of $M s p$ I in the gene, and the normal and mutant sequences. The one-base substitution of $\mathrm{nt} 508 \mathrm{C} \rightarrow \mathrm{T}$ caused the amino acid change of R170W 
a

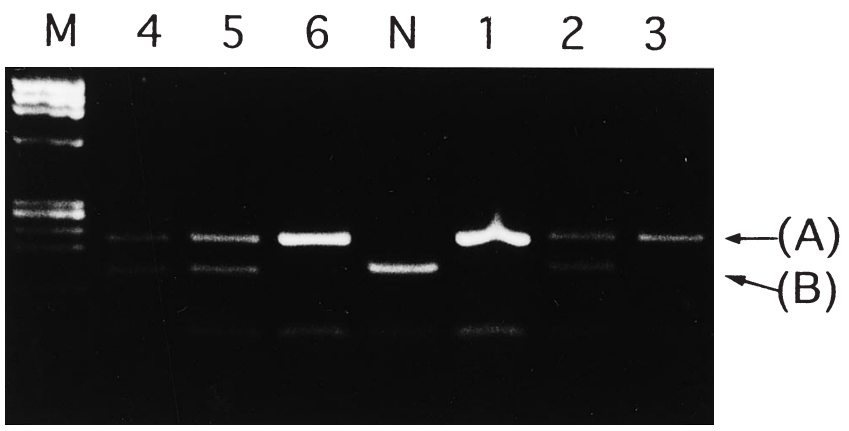

b

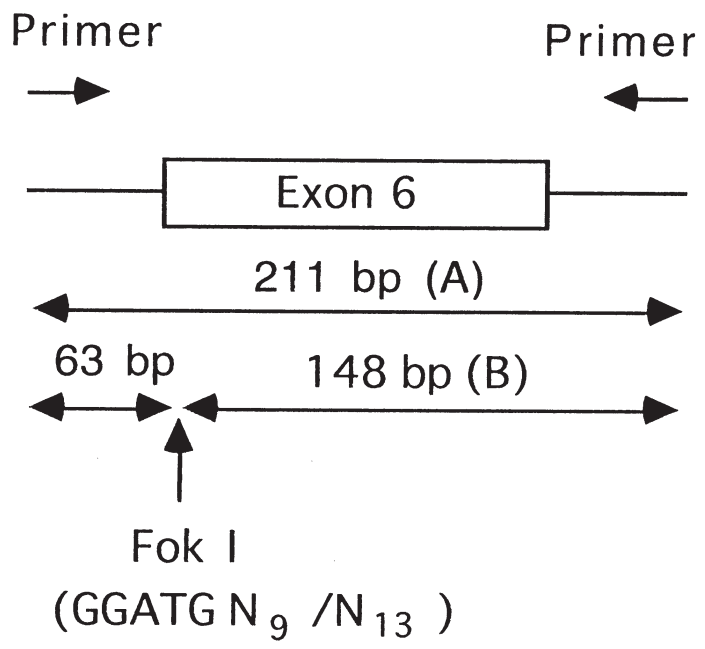

Normal: tacag GAT GTC ATG

Mutant: tacat GAT GTC ATG

Fig. 4a,b Screening of the major mutation; exon 6 and the flanking regions were amplified with the primers $5^{\prime}$-TGAAACCGGAGAGACTGTGATG-3' (sense) and 5'-GCCACAGCCAGATTCAGACATTG-3' (antisense), and then digested by Fok I. The PCR product derived from the normal allele was cut into two fragments, of $148 \mathrm{bp}$ (band $B$ ) and $63 \mathrm{bp}$ (not visible) by Fok I, while the product from the mutant allele (IVS $5,-1 \mathrm{G} \rightarrow \mathrm{T}$ ) was resistant to Fok $I$ (band $A$, $211 \mathrm{bp}$ ). a Agarose gel electrophoresis of Fok I-digested PCR fragment. Lane M, Molecular size marker; lane N, normal control; lanes 16 , subjects 1-6. Subjects 2, 4 and 5 were heteroallelic, and subjects 1,3 , and 6 were homoallelic for IVS $5,-1 \mathrm{G} \rightarrow \mathrm{T}$. b Schematic illustration of the restriction site of Fok I in the gene and the sequences of the normal and the mutant alleles. Asterisk indicates the mutation

$\mathrm{G} \rightarrow \mathrm{T}$ in the glucose-6-phosphatase gene in patients with glycogen storage disease type Ia (accounting for $90 \%$ of the mutant alleles) (Kajihara et al. 1995), the IVS $3,-1 \mathrm{G} \rightarrow \mathrm{C}$ of the $X P A$ gene in patients with xeroderma pigmentosum type A (accounting for $80 \%$ of the mutant alleles) (Satokata et al. 1990), and the IVS $5,-1 \mathrm{G} \rightarrow \mathrm{T}$ in the $\beta$ hexosaminidase $\alpha$ gene (accounting for $80 \%$ of the mutant alleles) (Tanaka et al. 1993) are examples. a
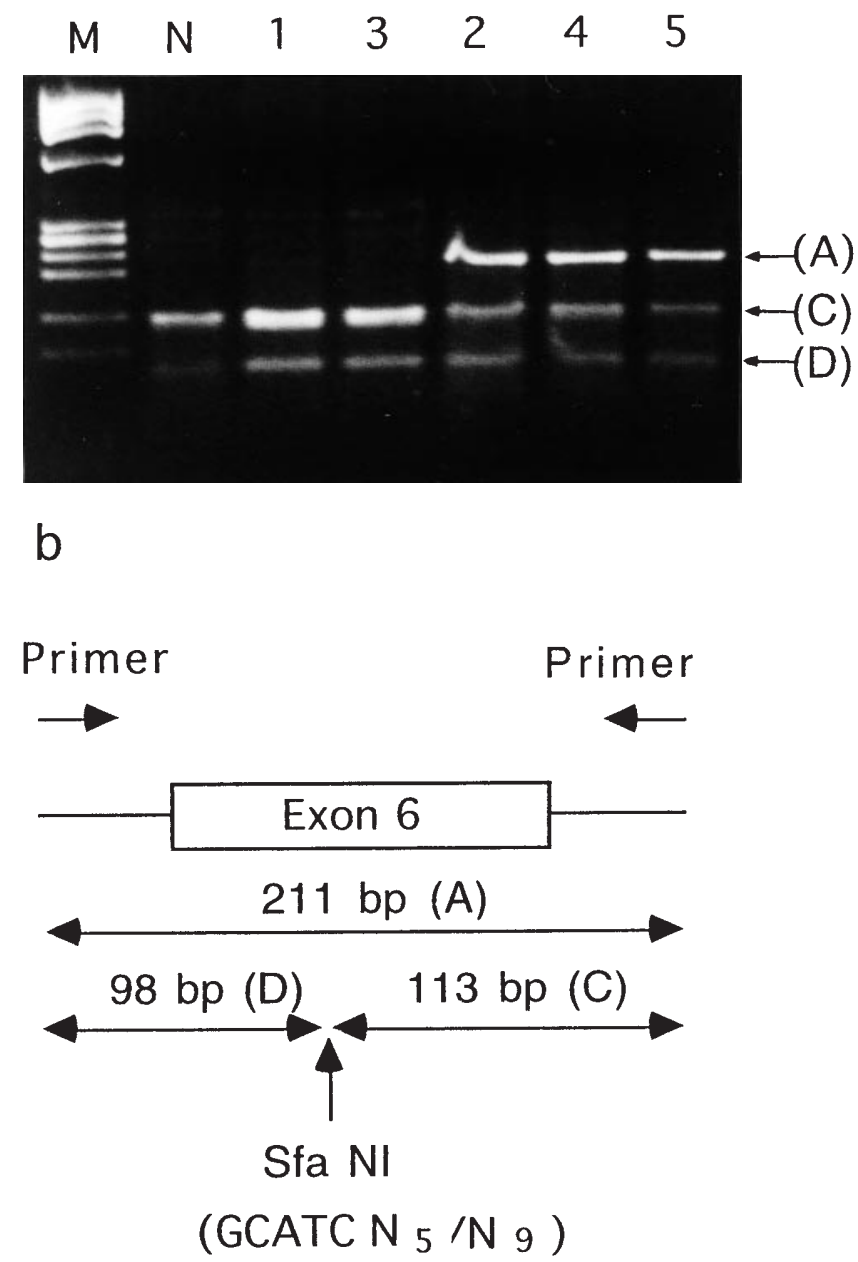

Fig. 5 Screening of the second most common mutation. The same region was amplified with the same primers as those described in Fig. 4, legend then digested by $S f a$ NI. The PCR product derived from the normal allele was cut into two fragents, of $113 \mathrm{bp}$ (band $C$ ) and $98 \mathrm{bp}$ (band $D$ ) by $S f a$ NI, while the product from the mutant allele (nt613C deletion) was resistant to $S f a$ NI (band $A, 210 \mathrm{bp}$, one base deleted). a Agarose gel electrophoresis of $S f a$ NI-digested PCR fragment. Lane $M$, Molecular size marker; lane $N$, normal control; lanes $1-5$, subjects $1-5$. Subjects 2, 4, and 5 were heterozygous for the deletion of nt613C. b Schematic illustration of the restriction site of Sfa NI in the gene and the sequences of the normal and the mutant alleles. Asterisk indicates the mutation

The deletion of $n t 613 \mathrm{C}$ is the first mutation, other than IVS $5,-1 \mathrm{G} \rightarrow \mathrm{T}$, found in Japan in more than single unrelated patients. These two most common mutations account for $85 \%$ of the mutant alleles causing infantile Tay-Sachs disease in Japan. Therefore, it is important to screen individuals at risk for these mutations. The procedure described in this report takes advantage of two factors; the relatively close locations of the two mutations in the gene and the 
altered restriction maps in both alleles. Thus, a single PCR followed by digestion with appropriate restriction enzymes provides a simple, rapid, and effective screening procedure. For families carrying either of these mutations, the procedure is applicable for unambiguous prenatal diagnosis.

Both of the two novel mutations reported in the this study result in the severe infantile form of the disease. This is consistent with the prediction that neither of the mutant alleles produces enzyme protein with residual catalytic function. The deletion of $n t 613 \mathrm{C}$ appears to generate no detectable mRNA. IVS 5, +1G $\rightarrow$ A abolishes the consensus sequence for splicing. The missense mutation of R170W has been reported in an Italian patient (Akli et al. 1993) and a French Canadian patient (Fernandes et al. 1992) with the severe infantile form of the disease. As this missense mutation is at the $\mathrm{CpG}$ sequence, it may occur independently. Another mutation at the same codon, R170Q, which is also at $\mathrm{CpG}$ and causes the severe infantile form of the disease, has been reported in a Japanese patient (Nakano et al. 1990) and a Scottish patient (Akli et al. 1993).

Acknowledgments We thank Dr. K. Inui (Osaka University), Dr. K. Sukegawa (Gifu University), Dr. K. Naritomi (Ryukyu University, Okinawa), and Dr. H. Sakuraba (Tokyo Metropolitan Institute of Medical Science) for providing the cultured skin fibroblasts from patients with Tay-Sachs disease. We also thank Professor Kunihiko Suzuki (University of North Carolina, Chapel Hill, NC, USA) for his critical readings of the manuscript and his many helpful suggestions.

This work was supported by Grants from the Department of Education, Japan (AT-06670818 and AT-08670905).

\section{References}

Akli S, Chomel J-C, Lacorte J-M, Bachner L, Poenaru L, Kahn A (1993) Ten novel mutations in the HEXA gene in non-Jewish TaySachs patients. Hum Mol Genet 2: 61-67

Arpaia E, Dumbrille-Ross A, Maler T, Neote K, Tropk M, Troxel C, Stirling JL, Pitts JS, Bapat B, Lamhonwah AM, Mahuran DJ,
Schuster SM, Clarke JTR, Lowden JA, Gravel RA (1988) Identification of an altered splice site in Ashkenazi Tay-Sachs disease. Nature 333: $85-86$

Fernandes M, Kaplan F, Nacowicz M, Prence E, Kolodny E, Kaback M, Hechtman P (1992) A new Tay-Sachs disease B1 allele in exon 7 in two compound heterozygotes each with a second novel mutation. Hom Mol Genet 1: 759-761

Kajihara S, Matsuhashi S, Yamamoto K, Kido K, Tsuji K, Tanae A, Fujiyama S, Itoh T, Tanigawa K, Uchida M, Setoguchi Y, Motoyama M, Mizuta T, Sakai T (1995) Exon redefication by a point mutation within exon 5 of the glucose-6-phosphatase gene is the major cause of glycogen storage disease type Ia in Japan. Am J Hum Genet 57: $549-555$

Myerowitz R (1988) Splice junction mutation in some Ashkenazi Jews with Tay-Sachs disease: Evidence against a single defect within this ethnic group. Proc Natl Acad Sci USA 85: 3955-3959

Myerowitz R (1997) Tay-Sachs disease-causing mutations and neutral polymorphisms in the Hex A gene. Hum Mut 9: 195-208

Myerowitz R, Hogikyan ND (1987) A delation involving alu sequences in the $\beta$-hexosaminidase $\alpha$-chain gene of French Canadians with Tay-Sachs disease. J Biol Chem 262: 15396-15399

Myerowitz R, Costigan FC (1988) The major defect in Ashkenazi Jews with Tay-Sachs disease is an insertion in the gene for the $\alpha$-chain of $\beta$-hexosaminidase. J Biol Chem 263: 18587-18589

Nakano T, Nanba E, Tanaka A, Ohno K, Suzuki Y, Suzuki K (1990) A new point mutation within exon 5 of $\beta$-hexosaminidase $\alpha$ gene in a Japanese infant with Tay-Sachs disease. Ann Neurol 27: 465-473

Ohno K, Suzuki K (1988) A splicing defect due to an exon-intron junctional mutation results in abnormal $\beta$-hexosaminidase $\alpha$ chain mRNAs in Ashkenazi Jewish patients with Tay-Sachs disease. Biochem Biophys Res Commun 153: 463-469

Satokata I, Tanaka K, Miura N, Miyamoto I, Satoh Y, Kondo S, Okada Y (1990) Characterization of a splicing mutation in group A xeroderma pigmentosum. Proc Natl Acad Sci USA 87: 9908-9912

Shambrook J, Fritsch EF, Maniatis T (1989) Molecular Cloning: A laboratory manual, 2nd edn. Cold Spring Harbor Laboratory Press, Cold Spring Harbor, NY

Tanaka A, Sakuraba H, Isshiki G, Suzuki K (1993) The major mutation among Japanese patients with infantile Tay-Sachs disease: A G-to-T transversion at the acceptor site of intron 5 of the $\beta$-hexosaminidase $\alpha$ gene. Biochem Biophys Res Commun 192: 539-546

Tanaka A, Sakazaki H, Murakami H, Isshiki G, Suzuki K (1994) Molecular genetics of Tay-Sachs disease in Japan. J Inher it Metab Dis 17: 593-600

Triggs-Raine BL, Akerman BR, Clarke JTR, Gravel RA (1991) Sequence of DNA flanking the exons of the HEXA gene, and identification of mutations in Tay-Sachs disease. Am J Hum Genet 49: 1041-1054. 